\title{
Initial Experience of Video-Assisted Thoracic Surgery Lobectomy After Neoadjuvant Chemotherapy Plus Toripalimab in a Patient with Locally Advanced Non-Small Cell Lung Cancer: A Case Report
}

Wei Li

Weifang Medical University - Kuiwen Campus

Chunbo Zhai ( $\square$ zhaicbmd@gmail.com )

Weifang People's Hospital https://orcid.org/0000-0003-2320-3380

Jianpeng Che

Weifang Medical University - Kuiwen Campus

Weiqian Wang

Weifang Medical University - Kuiwen Campus

Bingchun Liu

Weifang Medical University - Kuiwen Campus

\section{Case report}

Keywords: Non-small cell lung cancer, Neoadjuvant immunotherapy, Video-assisted thoracic surgery, Toripalimab, Case report

Posted Date: July 13th, 2021

DOI: https://doi.org/10.21203/rs.3.rs-151057/v2

License: (9) This work is licensed under a Creative Commons Attribution 4.0 International License. Read Full License

Version of Record: A version of this preprint was published at Journal of Cardiothoracic Surgery on January 16th, 2022. See the published version at https://doi.org/10.1186/s13019-021-01741-2. 


\section{Initial experience of video-assisted thoracic surgery}

\section{lobectomy after neoadjuvant chemotherapy plus}

\section{toripalimab in a patient with locally advanced non-small cell}

\section{lung cancer: a case report}

Wei Li, Chunbo Zhai ${ }^{*}$, Jianpeng Che, Weiqian Wang, Bingchun Liu

*Correspondence: zhaicbmd@gmail.com

Department of Second Ward of Thoracic Surgery, The First Affiliated Hospital, Weifang Medical University (Weifang People's Hospital), 151 Guangwen Street, Weifang, China 261041.

Background: Immune checkpoint inhibitors were used for patients with advanced non-small cell lung cancer (NSCLC) more and more frequently and the effects were thrilling. Toripalimab as a new immune checkpoint inhibitor has been shown to be effective in patients with advanced NSCLC. However, data regarding the safety and feasibility of surgical resection after treatment with toripalimab for NSCLC remain scarce. Here, we present a case with locally advanced NSCLC that received video-assisted thoracic surgery (VATS) lobectomy after treatment with toripalimab in combination with chemotherapy.

Case presentation: A 62-year-old male patient with a history of coronary artery stenting operation for two times was found a $3.4 \times 3.2 \mathrm{~cm}$ cavity mass in the upper lobe of the left lung and enlarged left hilar and mediastinal lymph nodes. Pathological results identified squamous cell carcinoma. The patient was diagnosed with a locally advanced NSCLC and received VATS left upper lobectomy and lymph node dissection after neoadjuvant chemotherapy plus toripalimab for 3 cycles. The postoperative pathological results showed complete tumor remission. Short-term follow-up results were excellent, and long-term results remain to be revealed.

Conclusions: Our preliminary results showed that the use of neoadjuvant toripalimab and chemotherapy for the locally advanced NSCLC before surgical resection is safe and feasible.

Keywords: Non-small cell lung cancer, Neoadjuvant immunotherapy, Video-assisted thoracic surgery, Toripalimab, Case report 


\section{Background}

Toripalimab as a humanized immunoglobulin G4 monoclonal antibody targeting programmed cell death protein 1 (PD-1) was approved for the treatment of unresectable or metastatic melanoma after the failure of previous systemic therapy in China on December $17^{\text {th }}, 2018^{1}$. A phase 1 trial characterized the encouraging antitumor activity and a controllable safety profile of toripalimab in patients with advanced NSCLC ${ }^{2}$. However, data regarding the safety and feasibility of surgical resection after treatment with toripalimab in combination with neoadjuvant chemotherapy remain scarce. We recently completed a VATS lobectomy for a patient with locally advanced NSCLC after treatment with toripalimab in combination with docetaxel and cisplatin. We expect that this case report will provide initial insights into the efficacy, safety, and impact of toripalimab on surgery.

\section{Case presentation}

A 62-year-old male with a chief complaint of bloating visited our hospital on May $29^{\text {th }}, 2020$. The patient with an 80 pack-year history of smoking underwent coronary artery stenting operation two times (One was on August 10 ${ }^{\text {th }}, 2017$, the other one was on October $1^{\text {st }}$ of 2017) due to coronary atherosclerotic heart disease. This patient has been treated with Plavix, aspirin, rosuvastatin and isosorbide mononitrate.

During physical examination, no obvious abnormal breath sounds in the chest or abnormal cervical lymph nodes were found. An enhanced computed tomography (CT) of the chest and positron emission tomography-computed tomography (PET-CT) showed a $3.4 \times 3.2 \mathrm{~cm}$ cavity mass in the upper lobe of the left lung and enlarged left hilar and mediastinal lymph nodes, with the largest node being about $2.6 \times 2.8 \mathrm{~cm}$ (Figure 1A, B). There were no obvious metastases based on magnetic resonance imaging of the brain and PET- CT of the abdomen and bone. Analysis using a fiber optic bronchoscope identified a tumor which blocked the opening of the proper segment of the left upper lobe. The pathological result by biopsy confirmed squamous cell carcinoma (Figure 1C).

Measurements of serum tumor markers showed elevated level of cytokeratin 19 fragment (Cyfra21-1: $4.77 \mathrm{ng} / \mathrm{mL}$, reference: $\leq 3.3 \mathrm{ng} / \mathrm{mL}$ ), neuron-specific enolase (NSE: $19.60 \mathrm{ng} / \mathrm{mL}$, reference: $\leq 15.2 \mathrm{ng} / \mathrm{mL}$ ), and squamous cell carcinoma antigen (SCCA: $2.58 \mathrm{ng} / \mathrm{mL}$, reference: $\leq 2.5 \mathrm{ng} / \mathrm{mL}$ ). The results were negative for serum carbohydrate antigen 125 (CA125), carcinoembryonic antigen (CEA) and vascular endothelial growth factor (VEGF). Immunohistochemical staining showed positive staining for CK5/6, P40 and PD-L1 (+80\% tumor cells), and negative staining for TTF-1, PD-1 and weakly positive staining for ALK-Ventana. Molecular analysis indicated no EGFR mutation or ROS1 fusion.

Combined with the patient's chest enhanced CT, bronchoscopy, and PET-CT conditions, the patient was diagnosed with a locally advanced NSCLC. On one hand, left pneumonectomy may be necessary if direct surgery was performed. However, the patient has a history of two times' coronary artery stenting operations which made the surgical risk of left pneumonectomy very high. On the other hand, it was very likely that lobectomy alone may not achieve complete microscopic resection (R0). It was 
therefore recommended to give neoadjuvant chemotherapy plus toripalimab for 3 cycles after lung cancer multiple-disciplinary team (MDT) assessment. If the tumor and lymph nodes are significantly reduced, then surgery will be given. If the tumor does not shrink, radiotherapy and chemotherapy will be given. The patient agreed with the treatment plan and signed an informed consent form.

From June $25^{\text {th }}, 2020$ to August $6^{\text {th }}, 2020$, the patient accepted 3 cycles' neoadjuvant chemotherapy (docetaxel $130 \mathrm{mg}$ and cisplatin $130 \mathrm{mg}$ ) plus immunotherapy (toripalimab $240 \mathrm{mg}$ ). the patient's chest enhanced CT on August $5^{\text {th }}$, 2020 showed that the tumor remission was significant (Figure 1D, E). After an MDT evaluation, surgical treatment was recommended after the third cycle's treatment. The patient's perioperative physical conditions and surgical indications were evaluated by enhanced CT of brain, chest and abdomen, and by coronary CT angiography, pulmonary function, and echocardiography.

The patient received the VATS left upper lobectomy and lymph node dissection on September $16^{\text {th }}, 2020$. The procedure was as follows: The thoracoscopic observation hole was at the $7^{\text {th }}$ intercostal space of the mid-axillary line, and the operative hole was at the $4^{\text {th }}$ intercostal space of the anterior axillary line and the $7^{\text {th }}$ intercostal space of the posterior axillary line. The branches of the lingual segmental artery, posterior segmental artery and left upper pulmonary vein were cut respectively by endoscopic linear cutting stapler after opening the fissure. Anterior apical artery and left superior lobar bronchus were densely connected by fibrotic lymph nodes. The left superior lobar bronchus was firstly cut with scissors at the bifurcation of lingual bronchus and proper bronchus. However, the adhesion of lymph nodes and blood vessels was still very dense. Thus vascular blocking tapes were put on the left pulmonary artery root and lower pulmonary artery respectively to avoid hemorrhage during operation (Figure 2A). The lymph nodes were carefully separated with scissors from the blood vessels after the vessels were blocked. The anterior apical artery was then cut with an endoscopic linear cutting stapler. The tumor and lung were placed in retrieval bags and removed through the front operation hole. Then the upper lobe bronchus stump was suspended with 3 silk threads and closed with an endoscopic linear cutting stapler (Figure 2B). Intraoperative frozen pathological examination showed that there was no residual tumor at the cut edge of the bronchus. Finally, the lymph nodes in groups $4,5,6,7,9,10$, and 11 were completely removed. Intraoperative blood loss was about $50 \mathrm{ml}$ without blood transfusion.

In the perioperative period, the patient's vital signs were stable, and there were no complications such as arrhythmia, thrombosis, pulmonary infection, and air leakage. The postoperative routine pathological results showed that the tumor had achieved complete remission (Figure $1 \mathrm{~F}$ ). The patient recovered well and was discharged on the $7^{\text {th }}$ day after surgery. Moreover, the patient received the $4^{\text {th }}$ cycle's chemotherapy and immunotherapy consistent with preoperative treatment plan on October $14^{\text {th }}, 202$

\section{Discussion and conclusions}

Previous study showed that patients with locally advanced (T2-T4) NSCLC or 
limited lymph nodal metastasis (N1, unilevel N2) need a multimodal therapy including surgery combined with adjuvant cisplatin duplet chemotherapy. However, complete microscopic resection (R0) and adjuvant chemotherapy only improves survival benefit by approximately $5 \%{ }^{3,4}$. In recent years, preoperative neoadjuvant chemotherapy combined with immunotherapy has achieved surprisingly high remission rates. The results of Checkmate159 trial showed that the major pathological response (MPR) of the nivolumab was $45 \%{ }^{5}$. The results of NCT02716038 showed that the MPR rate of atezolizumab combined with platinum-based dual regimen was up to $57 \%{ }^{6}$. The patient in our study was a locally advanced case because of the significantly enlarged and metastatic hilar lymph nodes. In this case, complete microscopic resection (R0) may not be achieved, and the long-term survival benefit may also be unsatisfied if operation was performed directly. Thus neoadjuvant chemotherapy plus immunotherapy for this patient was recommended by MDT assessment.

Toripalimab has been used to treat several kinds of unresectable or metastatic malignant tumors and the application of toripalimab in advanced NSCLC are increasingly reported. Preliminary data suggested that its safety and effectiveness were encouraging ${ }^{7-10}$. In particular, a recent single-center, open-label, parallel-control, phase 1 trial (NCT03301688) demonstrated the high security and exciting antitumor activity of toripalimab in patients with advanced NSCLC ${ }^{2}$. The trial results suggested that patients with PD-L1 expression of more than $50 \%$ could probably achieve long-term survival benefits and lasting response. In this case report, we presented some initial results about the surgical outcomes of VATS lobectomy after combined application of toripalimab with chemotherapy. Our preliminary results showed that there was a good pathological response and no apparent adverse events observed in the case ${ }^{11-13}$. The use of neoadjuvant chemotherapy and toripalimab for the locally advanced NSCLC before surgical resection was found to be safe and feasible. Our results suggest that toripalimab might be a promising treatment option for locally advanced lung cancer patients who have high expression of PD-L1. More studies are needed to further explore the potential role or effects of toripalimab in neoadjuvant combinations for resectable NSCLC.

Previous studies have shown that some patients still have residual tumors in radiology after receiving immunotherapy despite that the pathological remission has actually occurred ${ }^{12,14}$. Similarly, in this case, the preoperative enhanced CT showed that there are still a small number of residual tumors and lymph nodes after 2 cycles' neoadjuvant toripalimab immunotherapy in combination with cisplatin duplet chemotherapy. However, the postoperative pathological results showed that the tumor and lymph nodes achieved complete remission. These results suggested that traditional response criteria used to evaluate the effects of cytotoxic chemotherapy may be not suitable for checkpoint inhibitors ${ }^{15}$. Therefore, surgical resection is still an important option for the patients with residual tumors and/or lymph nodes in radiology.

Dense fibrosis as a result of an excellent response to immunotherapy may increase the difficulty of surgery and even lead to conversion to thoracotomy from 
thoracoscopy ${ }^{12}$. During the operation, it was found that the residual tumor tissues, lymph nodes, upper lobe bronchus and pulmonary artery of this patient were densely adhered, and the normal interstitial space disappeared. In this case, it was difficult to complete the operation with conventional thoracoscopic techniques. To solve this issue, a novel strategy was designed: the bronchus was cut with scissors at the bifurcation of the upper lobe lingual and proper bronchus after the left upper pulmonary vein and branches of pulmonary artery were treated first. Thus the relationship between the lymph nodes and the pulmonary artery can be better exposed, Scissors were then used to push the lymph nodes to the distal end of the pulmonary artery after the blocking tape temporarily blocked the left pulmonary artery stem and the lower pulmonary artery. Finally, the bronchial stump was closed with an endoscopic linear cutting suture device. The advantage of this strategy was that the tumor and lymph node can be fully assessed under the premise of ensuring safety. The relationship between trachea and pulmonary artery not only achieves the purpose of $\mathrm{R} 0$ resection, but also avoids hemorrhage and transfer to thoracotomy. Therefore it is a better method for VATS lung resection after immunotherapy.

Some limitations were unavoidable in this study. First, only one case was reported in this study. Our conclusions need to be further validated with more patient samples in the future. Second, since the main purpose of this study was to investigate the effect of toripalimab on perioperative period, long-term follow-up of patients was insufficient, and long-term follow-up of patients receiving toripalimab will be needed in future studies to assess overall survival and disease-free survival. In addition, lots of questions remain to be answered, such as how to determine preoperative complete remission of the tumors and whether surgery is necessary for these complete remission patients.

In conclusion, this case report suggested that toripalimab may be a promising choice for locally advanced NSCLC patients. Although surgery is challenging after immunotherapy, for the patients with resectable opportunity following immunotherapy, the VATS operation may deserve a try after temporarily blocking the pulmonary blood vessels to ensure the safety in operation.

\section{Abbreviations}

NSCLC: Non-small cell lung cancer; VATS: Video-assisted thoracic surgery; PD-1: Programmed cell death protein 1; CT: Computed tomography; PET-CT: Positron emission tomography-computed tomography; CA125: Carbohydrate antigen 125; CEA: Carcinoembryonic antigen; VEGF: Vascular endothelial growth factor; MDT: Multiple-disciplinary team; MPR: Major pathological response.

\section{Acknowledgements}

The authors thank the patient reported in this manuscript.

\section{Authors' contributions}

W. L. drafted the manuscript and C. Z. revised it. J. C., W. W. and B. L. acquired all figures. All authors read and approved the final manuscript for submission. 


\section{Funding}

The authors gratefully acknowledge funding support via Scientific Research Project of Health Commission of Weifang (No. wfwsjs-2018-096).

\section{Availability of data and materials}

Not applicable.

\section{Ethics approval and consent to participate}

This study was conducted with the approval at The First Affiliated Hospital, Weifang Medical University (Weifang People's Hospital). The patient in this study signed an informed written consent form.

\section{Consent for publication}

Not applicable

\section{Competing interests}

All authors declare no conflict of interest.

\section{References}

1. Liu H, Guo L, Zhang J, et al. Glycosylation-independent binding of monoclonal antibody toripalimab to FG loop of PD-1 for tumor immune checkpoint therapy. mAbs. 2019;11(4):681-690.

2. Wang Z, Ying J, Xu J, et al. Safety, Antitumor activity, and pharmacokinetics of toripalimab, a programmed cell death 1 inhibitor, in patients with advanced non-small cell lung cancer: a phase 1 trial. JAMA Netw Open. 2020;3(10):e2013770.

3. Goldstraw P, Ball D, Jett JR, et al. Non-small-cell lung cancer. Lancet. 2011; 378(9804): 1727-1740.

4. Arriagada R, Dunant A, Pignon JP, et al. Long-term results of the international adjuvant lung cancer trial evaluating adjuvant Cisplatin-based chemotherapy in resected lung cancer. J Clin Oncol. 2010; 28(1): 35-42.

5. Forde PM, Chaft JE, Smith KN, et al. Neoadjuvant PD-1 blockade in resectable lung cancer. NEJM. 2018; 378(21): 1976-1986.

6. Shu CA, Gainor JF, Awad MM, et al. Neoadjuvant atezolizumab and chemotherapy in patients with resectable non-small-cell lung cancer: an open-label, multicentre, single-arm, phase 2 trial. Lancet Oncol. 2020;21(6):786-795.

7. Lu M, Zhang P, Zhang Y, et al. Efficacy, safety, and biomarkers of Toripalimab in patients with recurrent or metastatic neuroendocrine neoplasms: a multiple-center phase Ib trial. Clin Cancer Res. 2020;26(10):2337-2345.

8. Yang J, Dong L, Yang S, et al. Safety and clinical efficacy of toripalimab, a PD-1 mAb, in patients with advanced or recurrent malignancies in a phase I study. Eur J Cancer. 2020;130:182-192.

9. Sheng X, Yan X, Chi Z, et al. Axitinib in combination with Toripalimab, a humanized immunoglobulin G4 monoclonal antibody against programmed cell death-1, in patients with metastatic mucosal melanoma: an open-label phase IB trial. J Clin Oncol. 2019;37(32):2987-2999.

10. Qiu H. Safety and efficacy of toripalimab in advanced gastric cancer: A new clinical trial bringing 
hope for immunotherapy in gastric cancer. Cancer Commun. 2020;40(4):194-196.

11. Eichhorn F, Klotz LV, Bischoff H, et al. Neoadjuvant anti-programmed Death-1 immunotherapy by Pembrolizumab in resectable nodal positive stage II/IIIa non-small-cell lung cancer (NSCLC): the NEOMUN trial. BMC cancer. 2019;19(1):413.

12. Bott MJ, Yang SC, Park BJ, et al. Initial results of pulmonary resection after neoadjuvant nivolumab in patients with resectable non-small cell lung cancer. J Thorac Cardiovasc Surg. 2019;158(1):269-276.

13. Bott MJ, Cools-Lartigue J, Tan KS, et al. Safety and feasibility of lung resection after immunotherapy for metastatic or unresectable tumors. Ann Thorac Surg. 2018;106(1):178-183.

14. Chaft JE, Hellmann MD, Velez MJ, Travis WD, Rusch VW. Initial experience with lung cancer resection after treatment with T-cell checkpoint inhibitors. Ann Thorac Surg. 2017;104(3):e217-e218.

15. Wolchok JD, Hoos A, O'Day S, et al. Guidelines for the evaluation of immune therapy activity in solid tumors: immune-related response criteria. Clin Cancer Res. 2009;15(23):7412-7420. 


\section{Figures:}
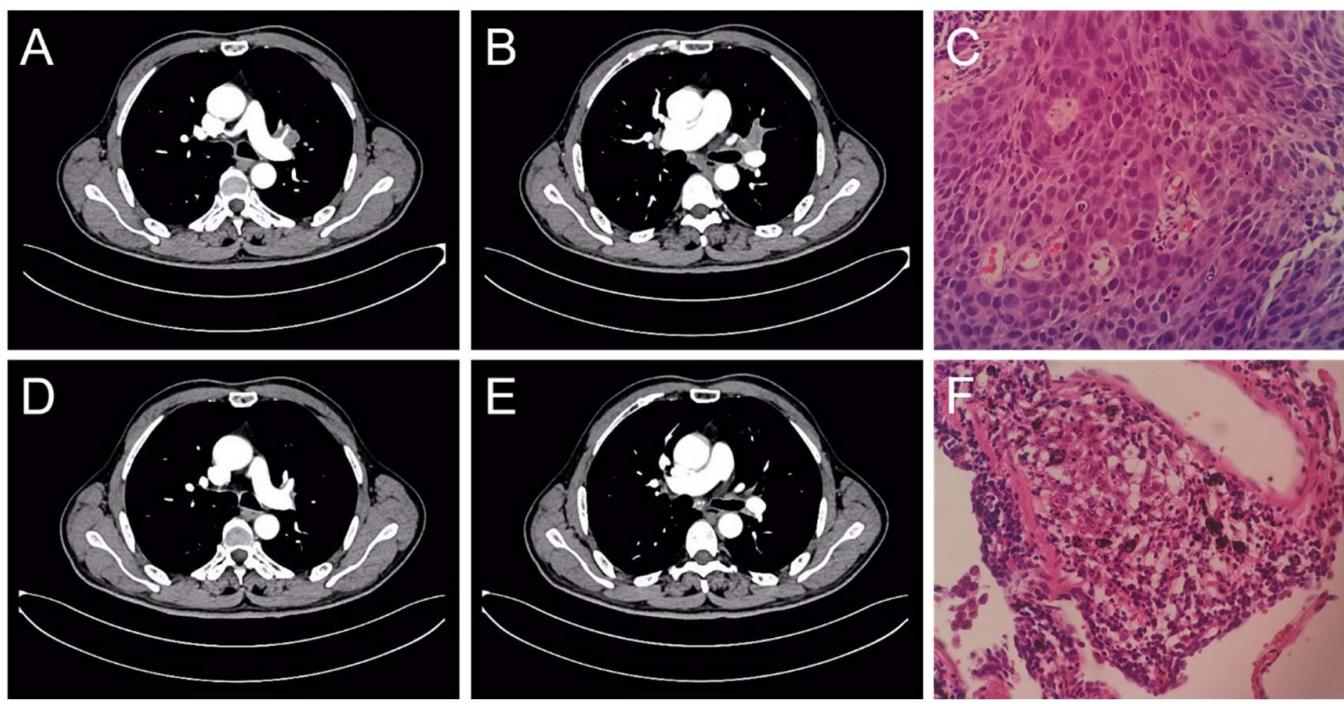

Figure 1. A and $\mathbf{B}$ are CT images before the treatments of neoadjuvant chemotherapy plus toripalimab. $\mathbf{C}$ is pathological image before the treatments. $\mathbf{D}$ and $\mathbf{E}$ are CT images after 3 cycles' treatments of neoadjuvant chemotherapy plus toripalimab. $\mathbf{F}$ is pathological image after the treatments of neoadjuvant chemotherapy plus toripalimab followed by VATS lobectomy.
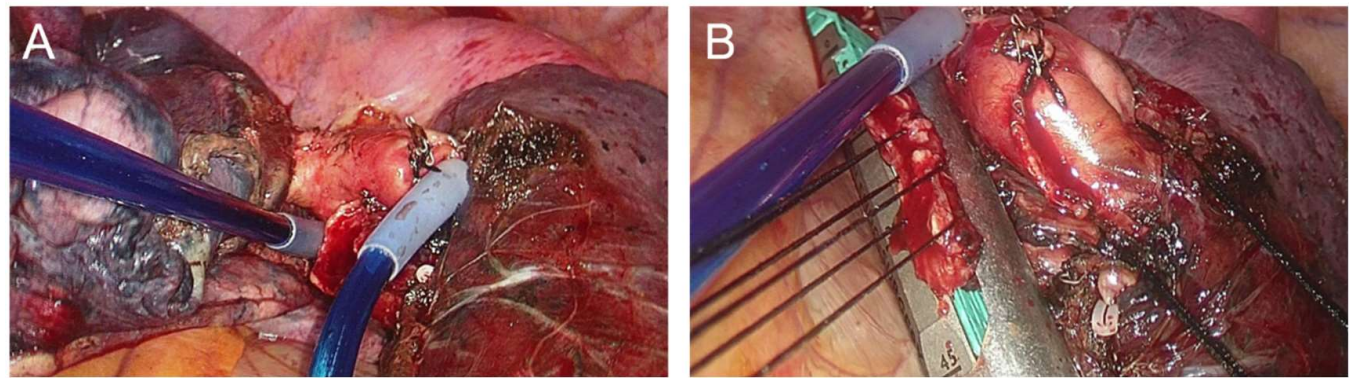

Figure 2. In A, vascular blocking tapes were put on the left pulmonary artery root and lower pulmonary artery respectively. In $\mathbf{B}$, the upper lobe bronchus stump was suspended with 3 silk threads and closed with an endoscopic linear cutting stapler. 


\section{Supplementary Files}

This is a list of supplementary files associated with this preprint. Click to download.

- CAREchecklist2021Zhai.pdf 